\title{
Los valores, la gestión y el desempeño de las pymes del sector industrial de Bogotá
}

\section{Values, management and performance of}

SMEs in the Bogota industry

Jorge Gámez-Gutiérrez** Carolina Aguirre Garzón ${ }^{* * *}$ Andrés Sánchez De la Rosa ${ }^{\text {**te* }}$

Recibido: 9 de abril de 2020

Revisado: 20 de mayo de 2020

Aprobado: 29 de mayo de 2020

* Cómo citar: Gámez Gutiérrez, J., Aguirre Garzón, C. y Sánchez De la Rosa, A. (2020). Los valores, la gestión y el desempeño de las pymes del sector industrial de Bogotá. Revista CIFE: Lecturas de Economía Social, 22(37), 43-79. DOI: https://doi.org/10.15332/22484914/6040

* Ph. D. en ciencias empresariales y economista. Profesor titular de la Universidad de La Costa. Correo electrónico: jgamez@cuc.edu.co

*** M. Sc. en política social y economista. Profesora de la Universitaria Agustiniana. Correo electrónico: carolina.aguirre@uniagustiniana.edu.co

***** Mc. en política social y economista. Estudiante de doctorado Public policy and political economy en University of Texas. Correo electrónico: sancheza@javeriana.edu.co 


\section{Resumen}

La gestión de las pymes depende de las condiciones del emprendedor, sus características y valores, sus estrategias y su gestión (Blackman, 2003). El desempeño de las empresas, medido en las variaciones del beneficio económico, depende de los valores del emprendedor y estrategias frente a su empresa, así como del emprendedor como individuo, su comportamiento humano, antecedentes, entorno, objetivos, valores y motivaciones (McClelland, 1970; Gámez, 2013). La permanencia de la empresa es la productividad (Sanabria, 2003), y esta se puede explicar a través de los valores de los emprendedores como logro, autonomía, ambición, agresividad, igualdad, autoridad, lealtad, innovación, trabajo y honestidad. Con 383 pymes de la industria de Bogotá se prueba el modelo de Blackman. El desempeño expresado en obtención de beneficios por el desarrollo de la actividad empresarial en la industria bogotana está repartido entre quienes crecieron, no tuvieron cambios y quienes han tenido decrementos.

Palabras clave: emprendedor, pyme, industria, valores, desempeño.

Glasificación JEL: Q13, R12, O18.

\section{Abstract}

Management of SMEs depend on the conditions of the entrepreneur, their characteristics and values, their strategies and their management (Blackman, 2003). The performance of companies measured in the variations of economic benefit depend on the entrepreneur, his values and strategies in relation to his company, the entrepreneur as an individual, his human behavior, his background, his environment, objectives, values and motivations (McClelland, 1970; Gámez, 2013). The permanence of the company is productivity (Sanabria, 2003), and this can be explained through the values of entrepreneurs such as achievement, autonomy, ambition, aggressiveness, equality, authority, loyalty, innovation, work and honesty. With 383 sMEs in the Bogotá industry, the Blackman model is tested. The performance expressed in obtaining benefits for the development of business activity in the Bogota industry is divided among those who grew up, did not have changes and those who have had decreases.

Keywords: Entrepreneur, sMEs, industry, values, performance.

JEL Classification: Q13, R12, O18. 


\section{Introducción}

La prioridad de los gobiernos en el último decenio se ha enfocado en corregir el bajo crecimiento económico. Algunos indicadores tuvieron un comportamiento estable como la inflación - entre 3\% y 4\%-, intereses moderados, baja volatilidad de la tasa de cambio, crecimiento económico moderado - entre $2 \%$ y $3 \%$ - tasa de desempleo de un dígito y una percepción de las calificadoras de riesgo de grado de inversión (Asociación Nacional de Empresarios de Colombia [ANDI], 2018; Fundación para la Educación Superior y el Desarrollo [Fedesarrollo], 2019; Banco Mundial, 2019).

La competitividad del país ha tenido avances, que han sido medidos en el ranking de Doing Business del Banco Mundial, al pasar del puesto 76 en 2006 al 34 en 2015, año desde el que se ve un estancamiento. En el Índice Global de Competitividad (IGC) del Foro Económico Mundial (WEF, por sus siglas en inglés), Colombia pasó del puesto 61 entre 140 países en 2015 al puesto 66 entre 137 en 2017 y, con el reciente cambio metodológico, del puesto 57 entre 135 al 60 entre 140. En el Anuario de Competitividad Mundial del Institute for Management Development (IMD), el país descendió del puesto 51 en 2015 al 58 en 2018. Estas clasificaciones evidencian que el país no ha progresado en la posición relativa en América Latina, porque Colombia retrocede y otros países progresan, como es el caso de Uruguay y Perú (Consejo Privado de Competitividad [CPC], 2018; Departamento Nacional de Planeación [DNP], 2017; Consejo Gremial Nacional, 2018).

En primer lugar, la baja productividad se puede explicar en parte por escasez de talento técnico calificado acorde con las necesidades del sector productivo. En segundo lugar, la economía colombiana es aún cerrada por cuenta de barreras no arancelarias, engorrosos trámites de comercio exterior y un sistema tributario ineficiente. En tercer lugar, Colombia es débil en innovación empresarial (DNP, 2013, 2017; Botero-García, López-Castaño, Posada, Ballesteros-Ruiz, \& García-Guzmán, 2015; CPC, 2018). La fuente del crecimiento futuro está en la productividad, por lo que Colombia debe apostarle a un crecimiento fundamentado en el incremento de la productividad (DNP, 2017; Organización para la Cooperación y el Desarrollo Económicos [OCDE], 2017).

Durante el 2018, la industria manufacturera tuvo un desempeño favorable; entre enero y octubre de ese año, la producción aumentó 3,2\% y las ventas totales 3,5\%, frente a los datos del 2017. Los empresarios consideran que el desempeño de la producción estuvo cercano a $3,1 \%$, las ventas totales al 3,3\% y las ventas al mercado interno al 2,8\% en la Encuesta de Opinión Industrial Conjunta. Además, la utilización de la capacidad instalada fue de 79,8\% para el 2018, mientras que en el 2017 alcanzó 76,2\%. Respecto de la producción reportada, $87,6 \%$ calificó sus pedidos como altos o normales en 2018, mejor que en 2017 (79,6\%). Los inventarios de la producción manufacturera fueron altos para el $17,5 \%$ de los empresarios encuestados en el 2018, mientras que en 2017 las existencias 
ascendieron a 21,4\%. En general, los principales obstáculos de los empresarios durante el 2018 fueron la falta de demanda, el costo de materias primas, las estrategias agresivas de comercialización, el tipo de cambio, el contrabando, la infraestructura, los costos logísticos, la incertidumbre tributaria, el capital de trabajo, la legislación y la cartera (ANDI, 2019; Consejo Gremial Nacional, 2018; DNP, 2017; Corporación Financiera Colombiana [Corficolombiana], 2017; Banco de la República, 2018).

El mayor número de empresas en Bogotá se encuentra en las actividades de servicios personales y empresariales ( $47 \%$ ), comercio ( $32 \%$ ) e industria manufacturera $(12 \%)$. En el 2018, se crearon 7995 empresas industriales en Bogotá y la región; de ellas, 99,2\% corresponde a microempresas, $0,7 \%$, a pequeñas y $0,1 \%$, a medianas. En la misma zona se encontraban empleadas 4,3 millones de personas. Estos empleos se generan en las actividades de servicios (50,9\%), comercio $(27,7 \%)$, industria $(14,9 \%)$ y construcción (5,6\%); el 59,6\% del empleo es asalariado. Para el mismo año, la liquidación de empresas se concentró en tres sectores (82\%): servicios (48\%), comercio (34\%) e industria (10\%). Se liquidaron 2346 empresas de la industria, de ellas microempresas $(94,3 \%)$, pequeñas $(4,6 \%)$, medianas $(0,8 \%)$, grandes $(0,3 \%)$ (Cámara de Comercio de Bogotá, 2019). Las actividades que más aumentaron fueron suministro de electricidad, gas y agua $(14.7 \%)$, explotación de minas y canteras $(10.6 \%)$, industria manufacturera $(2.2 \%)$, servicios comunales, sociales y personales $(1.1 \%)$, construcción $(0.9 \%)$ e intermediación financiera (0.3\%) (ANDI, 2019; Banco de la República, 2019).

\section{El emprendedor y sus características}

El emprendedor es aquel individuo que asume la creación de empresas, aprovecha un hueco en un mercado, aprovecha los resultados de una investigación, utiliza I+D como ventaja competitiva y tiene capacidad de acción (McClelland \& Winter, 1970; Drucker, 1984; Guzmán \& Liñán, 2005; Pereira, 2007; González, 2007). El emprendedor es un innovador radical que hace procesos de cambios sociales y tiene carácter multidimensional (Schumpeter, 1947a); asume un proceso constante de innovación, valioso por sí mismo y no por sus resultados (Pereira, 2007); además, toma riesgos financieros, psicológicos y sociales, con el fin de obtener recompensas como la independencia, la satisfacción personal y el beneficio económico cuando haya lugar (Hisrich, Langan-Fox, \& Grant, 2005).

Las características de los emprendedores pueden ser tanto propias como atribuidas (Blackman, 2003). Por una parte, las características propias del emprendedor incluyen su forma de actuar, como ser visionario y con sentido común (Fontela, Guzmán, Pérez, \& Santos, 2006); habilidades para afrontar la incertidumbre (Illera \& Illera, 2005); creatividad (Ponti \& Ferrás, 2008); intuición, imaginación y optimismo (Cañadas, 1996; 
Nuevo, 2009); autoconfianza, independencia autonomía y liderazgo (Gibb, 1988); compromiso, responsabilidad y perseverancia (Nuevo, 1994); empeño, autoridad y autocontrol (Prat, 1986), y habilidad de detectar oportunidades que le permitan alcanzar sus objetivos (Sexton \& Bowman-Upton, 1987; González, 2007).

Las características atribuidas del emprendedor son el resultado de distintos factores: a) la edad, ya que existe una mayor propensión hacia el emprendimiento entre los 25 y 40 años (Mayer \& Goldstein, 1961; Shapero, 1982); b) el sexo como determinante de su desempeño (Davidsson, 1995; Reynolds, Bygrave, Autio, Cox, \& Hay, 2002; Global Entrepreneurship Monitor Colombia [GEM], 2017); c) las diferencias entre los valores de los emprendedores en distintas culturas (Collins \& More, 1970; Gasse, 1982). Las poblaciones de inmigrantes se caracterizan por una mayor propensión hacia el emprendimiento como medio de subsistencia (Davidsson, 1995). Colombia ha sido un país de baja inmigración, donde su aislamiento y baja mezcla con otros pobladores genera endogamia por consanguinidad y endogamia cultural que resiste la entrada de las culturas, otras visiones de mundo y otros valores (Yunis, 2006). Algunas investigaciones sugieren que el éxito de los emprendedores está relacionado con las familias cuyos padres han sido emprendedores y, por tanto, los valores han sido transmitidos entre generaciones familiares (Cooper \& Dunkelberg, 1981; Davidsson, 1995; Rosenblatt, 1985; Engle, Schlaegeol, \& Dimitriadi, 2011).

\section{La organización}

El proceso de creación de empresa, según Veciana (2001), incluye las fases de gestación, creación, lanzamiento y consolidación. La fase de gestación contempla la infancia y preparación profesional del emprendedor, junto a un suceso disparador, las condiciones favorables del entorno y la decisión de crear la empresa; la segunda fase es la creación, y comprende la búsqueda e identificación de la oportunidad, la creación de la solución, las redes sociales, la evaluación de la oportunidad y los pasos para crearla; la tercera fase es el lanzamiento con duración media de uno o dos años, e implica crear la organización, la búsqueda de recursos y el lanzamiento del bien o servicio; por último, la fase de consolidación puede durar entre dos y cinco años e implica asumir el éxito o fracaso.

Por su parte, Guzmán y Liñán (2005) describen tres fases en un empresario: la potencial, la naciente y la dinámica. González (2007) arranca desde la búsqueda de la idea, su análisis, la construcción del plan de empresa - proyecto empresarial- y su creación - puesta en marcha- De acuerdo con una publicación del Banco Interamericano de Desarrollo (BID, Kantis, 2004), hay tres etapas del proceso emprendedor que termina en creación de empresa: la gestación, la puesta en marcha y el desarrollo inicial. En la gestación intervienen la motivación y las competencias del emprendedor, la identificación 
de la oportunidad y la elaboración del proyecto; en la puesta en marcha se toma la decisión de iniciar la empresa y se accede a los recursos para empezar; en la etapa de desarrollo inicial del proyecto se introducen los bienes y servicios en el mercado y se gestiona la empresa durante los primeros años. Por otro lado, el consorcio GEM (2017) diferencia entre empresas nacientes o start ups, con duración menor a tres meses; empresas nuevas o baby business, con duración entre tres y 42 meses; y empresas establecidas o established business con más de 42 meses de funcionamiento.

Algunas empresas nacen y permanecen como pequeñas empresas o microemprendimientos, según las habilidades del emprendedor como espíritu innovador, la autoestima, la confianza en su propia fuerza y la capacidad para enfrentar adversidades (Mouján, 2006). Además, Marx (1984) asumió la empresa como centro de explotación, forma de apropiación del tiempo y del trabajo ajeno, concentración y apropiación de la renta de los obreros que, además, se concentra solo en la búsqueda de ganancias. La empresa es, entonces, el sitio donde la productividad del trabajo crece y se suprime todo lo que sea trabajo inútil. En síntesis, para Marx (1984), la función del capitalista-empresario es una función económica de la nación que le es confiada por la propiedad del capital, y su ganancia es una forma de sueldo. Agrega Luxemburg (2003) que la apropiación de la plusvalía requiere incrementos de producción de capital para generarla y conservar las ventajas de las grandes empresas.

\section{Los valores}

Los valores son creencias que unen a las personas y las comprometen, que se transmiten en el medio empresarial a los trabajadores para alcanzar una meta común (Blanchard \& O’Connor, 1997). Los valores compartidos permiten marcar el camino a seguir, todos los miembros de la organización sabrían qué se espera de ellos y fomentan el espíritu de grupo (Robbins \& Coulter, 2005). A juicio de Kotter y Heskett (1995), los valores compartidos se corresponden con los objetivos e intereses de una organización compartidos por la mayoría de las personas, y tienden a condicionar su actuación; con frecuencia persisten durante un largo periodo, aunque haya cambios en la composición del grupo. Agregan Ramírez, Noel y Cabello (1997) que los valores son el conjunto de enunciados que reflejan los principios fundamentales en los que actúa una organización. Ogalla (2005) consideran que los valores apoyan la misión y la visión de la organización además de ser parte de la cultura organizacional.

Las características propias y atribuidas se expresan en los valores y las expectativas (Blackman, 2003). Los valores escogidos en este documento son logro, autonomía, ambición, agresividad, igualdad, autoridad, lealtad, innovación, trabajo y honestidad. 
Logro. Uno de los marcos para conceptualizar la motivación del logro es la teoría del valor de la expectativa (Eccles \& Roeser, 2009; Wigfield \& Eccles, 2000, Atkinson, 1957. Según esta teoría, la motivación del logro depende de dos elementos: a) la expectativa de éxito como creencias de cómo les irá en una actividad, y b) las creencias de valor que describen hasta qué punto se valora una actividad (Wigfield, 1994). Trautwein, Wiegmann, Beutel, Kjer y Yeates (2012) informaron un efecto perjudicial del valor de la tarea cuando las creencias de expectativa eran bajas. El valor de una tarea tiene cuatro componentes (logro, valor intrínseco, utilidad y costo) y sus interacciones. El logro es un elemento de la cultura corporativa que promueve el alcance de uniformidad en los componentes de la organización (Rokeach, 1973), con influencia positiva en el compromiso y el rendimiento (Kang, Jeon, Lee, \& Lee, 2005) y facilita el logro empresarial (Lord, 2006).

Autonomía. Se entiende como la capacidad de autogobierno expresada en formas de actuar de forma independiente, responsable y con convicción. Es además la competencia mental y capacidad cognitiva para decidir en un momento determinado, por tanto, se asocia a la edad legal. El valor de la autonomía se refiere a derechos y libertad de tomar decisiones por uno mismo sin interferencia. Sin embargo, tener la libertad de autogobernarse no necesariamente significa que el individuo tendrá de manera simultánea la capacidad y la oportunidad de ejercer el derecho de autogobierno. Las personas con altos niveles de desarrollo de la autonomía son menos propensas a ser afectadas por la casualidad, las contingencias o la debilidad de la voluntad en el ejercicio del autogobierno.

En el espacio empresarial, la autonomía impulsa a las personas a emprender la creación de empresas para buscar autonomía (Carter, Simkins, \& Simpson, 2003), circunloquio resuelto con el análisis factorial; Blais y Toulouse (1990), a partir de un estudio de 14 países, examinan con análisis factorial los motivos de independencia que resumen como control del tiempo propio, enfoque propio del trabajo, trabajar con las personas que elige, ser el propio jefe y liderar en lugar de ser dirigido.

Ambición. Se refiere a las aspiraciones de los individuos con respecto al desempeño personal; por extensión, en las empresas se busca el mejor desempeño del negocio. Se explica como la buena voluntad de crecimiento (Davidsson, 1989), motivaciones empresariales (Chell, \& Allman, 2003), intenciones empresariales (Krueger \& Carsrud, 1993) y aspiraciones de crecimiento nuevo (Tominc \& Rebernik, 2007).

Con los estudios de Global Entrepreneurship Monitor, a comienzos del siglo XXI se empezó a indagar por el desempeño expresado en ingresos por ventas (Cassar, 2007) y tamaño esperado de la empresa (Edelman, Brush, Manolova, \& Greene, 2010). Además, se incluyó la variable crecimiento combinada con innovación o expectativas de exportación (Bosma \& Schutjens, 2007, 2009; Hessels, Van Gelderen, \& Thurik, 2008a). 
Hermans et al. (2015) recogen tres momentos de la ambición del emprendedor: el crecimiento al que se aspira - idealmente quiere-, el que se tiene intención —el que intenta lograr - y el que se expera — quiere lograr y depende de las oportunidades-. Alcanzarlo implica estrategias éticas e integridad para no afectar los valores de integridad y confianza del equipo de trabajo (McCann, Sparks, \& Kohntopp, 2017)

Agresividad. La competencia supone la lucha por lograr rendimientos superiores, que en las empresas supone empujar al rival - otra empresa u otras empresas - en la constante búsqueda de mantenerse por delante del otro. Una empresa en un entorno competitivo puede mantener posiciones sólidas con ganancias superiores al promedio compitiendo agresivamente, de manera proactiva y enérgica, comenzando y respondiendo a los ataques de los competidores — ver teoría de juegos - (Ferrier, Fhionniaoich, Smith, \& Grimm, 2002). La competitividad agresiva propicia la creatividad y la innovación a cambio de los métodos tradicionales de competencia (Lumpkin \& Dess, 1996).

La agresividad se expresa en la preparación de las empresas para adaptarse a los rápidos cambios en el mercado y al progreso tecnológico; esta se traslada a los empleados para que sean creativos y acepten riesgos, y se prepara la organización para la ambigüedad y la incertidumbre del mercado (Lohn, Reis, Ribeirete, \& Swirski, 2017). Dean, Thibodeaux, Beyerlein, Ebrahimi y Molina (1993) destacaron la importancia de la competitividad agresiva para el desempeño de la empresa estadounidense en el mercado global, donde la competitividad explicó considerablemente más variación $(37 \%)$ en el emprendimiento corporativo que en cualquier otra variable estratégica/estructural analizada. Sin embargo, otros estudios muestran que las empresas que presentan un declive están más motivadas por una competencia agresiva (Covin \& Slevin, 1989; Ferrier, Mac Fhionnlaoich, Smith, \& Grimm, 2002).

Igualdad. El valor de igualdad es principio normativo organizador de lo social, en el entendido de que las controversias sobre la igualdad tienen que ver en su mayoría con la aplicación de la igualdad como principio. La desigualdad se expresa en creación, gestión y obtención de resultados diferentes en las empresas. En el tejido empresarial es evidente que no todos los individuos son iguales y las empresas tampoco: las empresas pueden adaptar la contratación y gestión de recursos humanos, prácticas y políticas a través de medidas obligatorias y voluntarias; en las pymes parece ser más viable trabajar por la igualdad que las grandes empresas (International Labour Office, 2007).

En las empresas se pueden presentar posibles discriminaciones directas e indirectas de la dirección contra grupos específicos de personas dentro del lugar de trabajo (McEnrue, 1993; Cox \& Blake, 1991; Bartz et al., 1990; Ross \& Schneider, 1992; Hammond \& Kleiner, 1992). Las organizaciones pueden comunicar sus valores y objetivos de igualdad a sus empleados, y evaluarlos, para garantizar su participación, su compromiso y su apoyo a la gerencia (Paelmke \& Erwee, 2008). 
Autoridad. Fayol (2003) no menciona explícitamente el poder, pero trata de forma implícita una especie de "autoridad moral" al enumerar los elementos que permiten a los gerentes hacer una contribución distintiva: salud, inteligencia, cualidades morales, educación general y conocimiento administrativo. Taylor (2003) tenía una teoría implícita de la autoridad "ingenua": el jefe sabe mejor que nadie y debe analizar de manera científica lo que hacen sus subordinados, para luego darles las órdenes apropiadas. Para ejercer la autoridad, algunos se basan en control con el significado de sensación de poder. El concepto de autoridad hecho por Max Weber (1984) en los negocios muestra que a principios del siglo xx esta se basaba en los derechos de propiedad o en la posición gerencial. Aun hoy se ejerce como "dar órdenes", se espera de los trabajadores una obediencia ciega y, en consecuencia, se puede romper la iniciativa y desalentar la autosuficiencia (Djebarni \& Ebie, 2011).

Barney y Hansen (1994) sugieren que los valores y creencias de la organización pueden estar respaldados por sistemas internos de recompensa y compensación, junto con sistemas de toma de decisiones que reflejen la cultura. Esta confianza organizacional también se puede llamar confianza de rutina, y surge especialmente en relación con relaciones institucionalizadas a largo plazo. En la organización también existe una confianza general a nivel de la empresa, y eso se basa en la buena reputación o los recursos de la empresa.

Lealtad. Este valor se entiende como la confianza para cooperar, junto con la coerción y el interés propio (Nooteboom, Berger, \& Noorderhaven, 1997). En el ambiente empresarial se asume la lealtad como compromiso expresado en apego a la organización, porque cree en el valor organizacional, muestra deseos de contribuir al logro de los objetivos de la empresa y manifiesta ansias de pertenecer a la organización. El compromiso cumple un fin en la reducción del absentismo, la rotación y las mejoras en su desempeño (Wong, Wong, \& Ngo, 2002).

La lealtad de los empleados se promueve en la empresa y se expresa cuando los empleados creen en los objetivos de esta, aceptan los objetivos como propios, trabajan para el bienestar común y desean permanecer en ella. El apego a la empresa se desarrolla en las circunstancias de identificación con ella. La lealtad de un empleado puede expresarse a través del sentimiento de la persona de pertenecer a la empresa, hacer equipo con compañeros de trabajo, permanecer en la empresa, tener la voluntad de hacer su trabajo y difundir información sobre el buen nombre de la organización (Varona, 2002).

Innovación. La innovación como expresión de la creatividad es importante para los individuos. Esta hace parte del desarrollo organizacional porque permite integrar la creatividad y el intercambio de conocimientos. Gracias a ella se generan procesos creativos de análisis del entorno, identificación de oportunidades y generación de ideas, y permite la optimización de objetivos, equipos y recursos (Vrontis \& Thrassou, 2013). 
Se pretende que en la empresa la innovación permita - junto con los empleados — la selección de proyectos, reducción de riesgos y tiempo de comercialización (Stoycheva \& Lubart, 2001). La innovación permite la exploración de espacios estratégicos para generar nuevas ideas con valor potencial, aprendizaje conjunto con empleados y clientes; además, facilita la concepción de la estrategia (Hatchuel \& Weil, 2003). La innovación se expresa en conocimiento generado en común por los miembros de una empresa y se aplica a las prácticas cotidianas (Nonaka \& Takeuchi, 2008; Nonaka \& Von Krogh, 2009; Felin et al., 2012).

Trabajo. El trabajo como valor refleja los esfuerzos para lograr la realización personal y profesional. El proceso de humanización se fundamenta en el trabajo como instrumento de humanización o deshumanización. En las organizaciones ocupa un lugar importante y permite conocer el grado de satisfacción o insatisfacción personal y profesional de los trabajadores (Bettinelli, Waskievicz, \& Erdmann, 2004).

El ambiente en la empresa afecta el alcance de objetivos y metas personales y organizacionales. Los trabajadores satisfechos con su trabajo son reflejo de la calidad del trabajo y ofrecen retroalimentación positiva a los clientes, lo que supone la humanización que surge de la satisfacción con el trabajo y el logro del clima organizacional y las adecuadas condiciones de trabajo (Backes, Koerich, \& Erdmann, 2007). La ética como uno de los factores más críticos en los negocios puede convertirse en obstáculos para el desarrollo de la ética; no obstante, la ética del trabajo ha demostrado ser un elemento necesario para el desempeño laboral (Salahudin, Alwi, Baharuddin, \& Halimat, 2016).

Honestidad. Zarate (2003) define el valor de la honestidad como lo propio de la naturaleza humana que hace referencia a la verdad y no hay cabida a la dualidad o el engaño. En la empresa, la integridad fue propuesta como componente integral del liderazgo transformacional por Burns (1978), con vínculos entre el liderazgo transformacional y resultados organizacionales (Lowe \& Gardner, 2000; Lowe, Kroeck \& Sivasubramanium, 1996; Avolio \& Bass, 1991; Bass, 1985).

Mayer et al (1995) propuso que la integridad se basa en valores morales percibidos por otros. Asimismo, Kouzes y Posner (2002) encontraron que aquellos individuos con honestidad generalmente practican lo que predican y harán lo que dicen que harán. Por su parte, Brenkert (2010) descubrió que la honestidad en los negocios se asocia a la promoción del comportamiento ético en las organizaciones. El valor de la honestidad está asociado con la percepción moral del comportamiento en situaciones de adversidad y desafío (Palanski, Gullen, Gentry, \& Nichols, 2015). Monga (2016) encontró que un significado adicional de integridad es consistencia frente a la adversidad. 


\section{Las prácticas gerenciales}

La creación y gestión de una empresa requieren que el emprendedor descubra el conocimiento útil y sus límites, además de usar la información para enfrentar las imperfecciones del mercado (Kirzner, 1986). La gerencia implica conocimiento técnico acompañado de habilidades blandas: creatividad, capacidad de trabajar en equipos multidisciplinarios y pensamiento asociativo para adquirir nuevos conocimientos rápidamente, y habilidades de comunicación (Avvisati, Jacotin, \& Vincent-Lancrin, 2013).

Guzmán y Santos (1999) recogen tres funciones del empresario: a) capitalista o financiera, destacada por fisiócratas, clásicos y marxistas, que llegaron a entender como uno solo al empresario y al capitalista; b) gerencial, propuesta por los economistas neoclásicos, marginalistas e institucionalistas, y que ejerce el empresario cuando dirige, organiza, gestiona, planifica o controla el funcionamiento de su empresa, y c) impulsora, planteada por economistas (Schumpeter, 1947; Knight, 1942) y no economistas (McClelland, 1961), referida a cualidades psicológicas y sociológicas del empresario, que debe decidir cuáles son las iniciativas que debe emprender. Por otra parte, quien ejerce la dirección de la empresa es quien más domina la capacidad administrativa; es decir, del obrero se espera la capacidad técnica, y de los mandos medios las demás funciones (Fayol, 2003). Además, propuso la diferenciación entre administración - función que asegura la gerencia - y gerencia - conducción de la empresa hacia su objetivo, tratando de sacar el mejor partido de los recursos que posee-

Los gerentes determinan la dirección de sus organizaciones, proporcionan liderazgo y deciden cómo utilizar los recursos para lograr las metas de las organizaciones (Drucker, 1954). Por lo tanto, la óptima gerencia se soporta en las funciones básicas de planeación organización, dirección y control (Nickels, McHugh, \& McHugh, 1997; Koontz \& Weihrich, 2003); los modelos gerenciales enfocados a generar políticas y administrar activos (Amit \& Zott, 2001; Magretta, 2002; Casadesus-Masanell \& Ricart, 2007); las habilidades gerenciales, personales, interpersonales y grupales (Whetten \& Cameron, 2011); las buenas prácticas (Delis \& Tsionas, 2018), y mejoras en sistemas de producción, prácticas de mercadeo, atención de las necesidades de sus clientes, sistema claro de premios y castigos al desempeño, consulta a expertos ajenos a sus empresa, entre otras (Blackman, 2003).

Rokeach (1973) y Robbins (1999) mostraron que las organizaciones pueden promover condiciones para que sus miembros se comporten influenciados por los valores que comparten la organización y sus miembros. McDonald y Gandz (1992) analizan las estrategias que se usan en las organizaciones para lograr la congruencia de los valores, entre ellas las acciones para reclutar y seleccionar candidatos congruentes con los valores de la organización, acciones para socializar entre esos empleados los valores deseados por 
la organización y acciones para modificar los valores que la organización requiere como respuesta a los cambios percibidos en su entorno más competitivo.

Delis y Tsionas (2018) consideran que la cuantificación de las prácticas gerenciales es indispensable para entender la dinámica organizacional, la economía, las finanzas y otras ciencias sociales. En este mismo sentido, los autores consideran que existe una dificultad en su medición debido a la escasez de datos e incluso a lo amplio del concepto. Los modelos que se aproximan a la medición en contextos industriales están dirigidos a la gran industria — véase el 2010 Manufacturing Survey Instrument (Centre for Economic Performance, 2015) — . Según Zucker (1986), la confianza organizacional puede provenir de la personalidad del propietario (pequeñas empresas) o de una estructura de decisión y cultura organizacional fuertemente centralizada, lo que hace que la organización interactúe regularmente de una manera particular y "confiable".

Amichai-Hamburger (2008) destaca, a partir de Siliceo (1997), los valores en la empresa, puesto que son los cimientos de "toda cultura organizacional [...] que conforman [...] el soporte filosófico del ser y quehacer de la empresa" (2008, p. 136). Entre esos resalta la calidad, comunicación, compromiso, educación, creatividad y redes de trabajo. Este autor afirma que los beneficios de la aplicación de los valores en la empresa, entre ellos la humanización de las relaciones y el sentido que otorgan a la vida laboral, posibilitan la cohesión de los grupos, aumentan la producción y calidad de los bienes y servicios y contribuyen a configurar la "personalidad" de la organización. Aryee, Budhwar y Chen (2002) consideran que la confianza en el líder se correlaciona con la confianza organizacional. El futuro de la organización depende de las condiciones de trabajo y el trato (Kiefer, 2005) o la inseguridad laboral (Wong, 2005), que pueden conducir a la desconfianza de los empleados en la organización. Whitener, Brodt, Korsgaard y Werner (1998) sugirieron que factores organizacionales como la estructura, las políticas y procedimientos de recursos humanos y la cultura organizacional afectarían las percepciones de confianza de los empleados.

Blackman (2003) propuso relaciones entre las características, los valores y las expectativas de los empresarios; entre sus valores y expectativas y las prácticas de gestión, y entre sus prácticas de gestión y el rendimiento empresarial en el contexto de las pymes en la industria (figura 1). El desempeño gerencial en una pyme se puede analizar desde varias perspectivas: el gerente es efectivo si el grupo que administra es efectivo (Elenkov, 2002; Riggio, Riggio, Salinas, \& Cole, 2003); otras personas consideran que el gerente es efectivo (Anderson, Salvucci, Ruane, Roads, \& Kanamitsu, 2008; Foti \& Hauenstein, 2007; $\mathrm{Ng}$, Ang, \& Chan, 2008), si el gerente se considera a sí mismo como gerente efectivo (Ng et al., 2008), si da un buen ejemplo de comportamiento y si puede convencer a las personas de que es un líder competente. 
Figura 1. Modelo teórico del desempeño de las pymes a partir de los valores y expectativas, las características y prácticas gerenciales del emprendedor

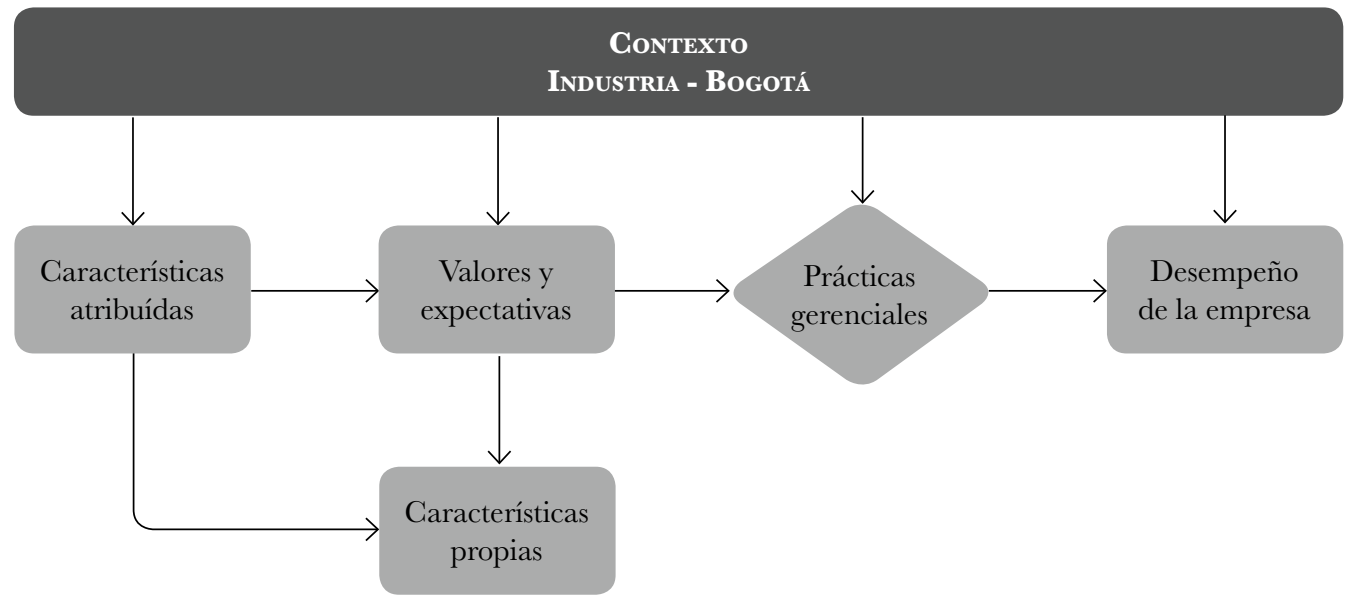

Fuente: elaboración propia con base en Blackman (2003).

El modelo del Centre for Economic Performance (2015) es valioso para optimizar la gestión de las empresas. Sin embargo, está diseñado para la gran empresa y no contempla la pyme. Por el contrario, en la tabla 1 el modelo de Blackman (2003) analiza en el emprendedor las características, valores y expectativas, así como los estilos gerenciales, y la empresa.

Tabla 1. Modelos de gestión de Centre for Economic Performance y Blackman

Centre for Economic Performance (2015)

a- Del gerente

Se miden las competencias de un gerente, solo es posible en grandes empresas no Pymes
Blackman (2003)

a- Del emprendedor Características propias

Características atribuidas

Valores y expectativas

Estilos gerenciales

\section{b- Organización}

Análisis de jerarquía en la empresa- aplicable solo en grandes empresas

\section{c- Propietarios}

No aplica para pymes, aunque gran parte del tejido empresarial es de empresas de familia

\section{d- Recursos humanos}


En este documento se analizan las pymes industriales de Bogotá y se consideran los atributos personales del emprendedor; además, incluye los valores, describe unos puntos básicos de la gestión y relaciona los resultados con otras variables basadas en las personas. Dentro de las limitaciones está la complejidad de la medición de valores y expectativas; además, las prácticas gerenciales tienen componentes subjetivos muy fuertes y se aborda en Bogotá.

\section{Marco referencial}

Lograr un desempeño organizacional sostenido a través del desarrollo de una fuerza laboral capaz se encuentra en el corazón mismo de la gestión del talento en Estados Unidos. Solo el 20\% de las organizaciones en ese país utiliza constantemente un proceso de incorporación sistemática para nuevos empleados en toda la organización; el 54\% no ofrece trabajo en red, tutoría o apoyo laboral por primera vez de 6 a 12 meses de empleo; el $45 \%$ informa que no pueden mantener un alto nivel de compromiso con las nuevas contrataciones durante su primer año. La mayoría de las empresas y sus equipos de gestión no son muy buenos en eso: más de la mitad (56\%) de los encuestados no sienten que su organización sea eficaz en el desarrollo de la capacidad de sus empleados, el desarrollo de capacidades ocurre esporádicamente, y con responsabilidad limitada. La mayoría de las organizaciones no creen que los gerentes tengan las capacidades necesarias para gestionar y desarrollar el talento. Los valores de la empresa no llegan en su totalidad a los empleados que no reciben suficiente orientación sobre cómo desarrollar sus habilidades y crecer en la organización.

Colombia se encuentra por debajo del promedio de América Latina y por debajo de México, Chile, Argentina y Brasil en prácticas gerenciales, según el Centre for Economic Performance (2015). Particularmente bajo es el desempeño de la gerencia en cuanto a gestión de operaciones. Esto se reafirma en el IMD World Talent Ranking 2017, en el que Colombia ocupa el puesto 55 de 63 economías (CPC, 2018; DNP, 2013; Institute for Management Development [IMD], 2018). Una de las debilidades de las empresas incluye bajas tasas de innovación debido a la pobre calidad de la gerencia (CPC, 2018), que no permite aprovechar las oportunidades de incorporar tecnología y gestionar los cambios necesarios para adaptarse a diversas formas de competencia (Consejo Gremial Nacional, 2018; DNP, 2013).

El Estado pretende incentivar la innovación con magros resultados: entre 2015 y 2017, se lograron once licenciamientos de tecnología provenientes de universidades; los Ph. D. se concentran en la academia más que en las empresas (88.9 de los investigadores), el Estado y las empresas vinculan pocos investigadores, y la evolución de solicitudes patentes muestra 2049 solicitudes en 2007 frente a 18552 en 2016 (CPC, 2018; DNP, 2013). 


\section{Metodología y resultados}

El diseño de la investigación se fundamenta en una metodología cuasi experimental y multifactorial. Por lo tanto, es cuantitativa y deductiva (Glaser \& Straus, 1967; Locke, 2001). Las relaciones propuestas entre las variables (Joreskog \& Sorborm, 1986) se pueden ver en la figura 1; en su orden, las variables que representan las características atribuidas impactarán las variables que representan características obtenidas; estas impactarán sobre las variables que representan los valores y expectativas de los emprendedores; las variables que representan características atribuidas impactarán los valores y expectativas de los emprendedores; las variables que representan los valores y expectativas impactarán sobre las prácticas gerenciales, y las variables que representan las prácticas gerenciales impactarán sobre los indicadores de desempeño de la empresa.

La investigación incluye información secundaria consignada en distintos informes de Cámara de Comercio, el Ministerio de Comercio, Industria y Turismo, la Secretaría de Hacienda, el Departamento Administrativo Nacional de Estadística (DANE) y Asociación Nacional de Empresarios de Colombia (ANDI), entre otros. Las fuentes primarias son las encuestas a 383 empresas de la industria (tabla 2) a partir de una base de datos del DANE, con 976987 registros de empresas de todo el país. En 2018, Bogotá y la región contaba con 487141 empresas de las cuales 58600 (12\%) están en la industria (Cámara de Comercio de Bogotá, 2017; Unipymes, 2019).

Tabla 2. Muestra por sectores, número y participación relativa

\section{International Standard Industrial Classification of All Economic Activities (ISIC)}

1300. Fabricación de tejidos de punto y ganchillo/ Fabricación de tapetes y alfombras para pisos/ Fabricación de cuerdas, cordeles, cables, bramantes y redes/ Fabricación de otros artículos textiles n.c.p.

1400. Fabricación de artículos de piel/ Fabricación de artículos de punto y ganchillo.

1511. Curtido y recurtido de cueros; recurtido y teñido de pieles

1512. Fabricación de artículos de viaje, bolsos de mano y artículos similares elaborados en cuero, y fabricación de artículos de talabartería y guarnicionería

1521. Fabricación de calzado de cuero y piel, con cualquier tipo de suela
Número

\section{Participación relativa}

41

$10.7 \%$
$5.0 \%$

$8.1 \%$

31

75

$19.6 \%$

68

$17.8 \%$ 
Revista CIFE / ISSN: 0124-3551 e-ISSN: 2248-4914 / Bogotá-Colombia / Vol. 22 N.o 37 / julio-diciembre 2020 / pp. $43-79$

$\longrightarrow$ Continuación

International Standard Industrial Classification of All Economic Activities (ISIC)
Número

47
Participación relativa

1600. Transformación de la madera y fabricación de productos de madera y de corcho, excepto muebles; fabricación de artículos de cestería y espartería

1800. Actividades de impresión y de producción de copias a partir de grabaciones originales

$18.8 \%$

2023. Fabricación de jabones y detergentes, preparados para limpiar y pulir; perfumes y preparados de tocador

2500. Fabricación de productos elaborados de metal, excepto maquinaria y equipo

Total
5

$1.3 \%$

25

383
$12.3 \%$

\section{Fuente: elaboración propia.}

El cuestionario está dividido en cuatro partes y tiene 45 preguntas: la primera parte recoge información sobre el emprendedor, país de procedencia, conformación de su núcleo familiar y grado de escolaridad; la segunda indaga sobre la información básica de la empresa tratando aspectos como año de creación, tipo de industria, conformación de la plantilla de empleados en relación con el número de familiares presentes en la empresa y algunos datos básicos sobre las actividades de planeación que hace la empresa; la tercera parte evalúa con distintas escalas la percepción que tiene el emprendedor respecto a temas como los objetivos empresariales, el éxito de su negocio, los valores familiares y empresariales; la cuarta parte examina el estilo de gestión del emprendedor y su desempeño.

Respecto de la caracterización de la muestra se encontró lo siguiente:

a) De los 383 emprendedores, 199 son hombres (52.0\%) y 184 mujeres (48.0\%). Cabe aclarar que la presencia de la mujer ha crecido en el mundo empresarial en los últimos diez años (Varela, Moreno, \& Bedoya, 2015).

b) Hay 382 colombianos y un venezolano, dos con padre extranjero (uno de Ecuador y uno de Venezuela), uno de madre venezolana. Al respecto, cabe mencionar que Colombia es un país de baja migración (Yunis, 2009).

c) Respecto de la forma como son propietarios de empresa, 105 la compraron (27.5\%), 219 la fundaron $(57.5 \%), 55$ la heredaron $(14 \%)$ y cuatro la adquirieron de otra forma $(1 \%)$.

La edad promedio de las empresas es 16.6 años (tabla 3). Son empresas consolidadas según GEM (2017), puesto que un emprendedor naciente es aquel que tiene una empresa de 0 a 3 meses, el nuevo empresario tiene una empresa de hasta 42 meses y el empresario establecido lleva más de 42 meses pagando salarios. 
Tabla 3. Edad de la empresa

\begin{tabular}{|lcc|}
\hline \multicolumn{1}{|c|}{ Edad de la empresa } & Número & Participación relativa \\
\hline Hasta 5 años & 49 & $12.8 \%$ \\
\hline Entre 6 y 10 años & 78 & $20.4 \%$ \\
\hline Entre 11 y 15 años & 72 & $18.8 \%$ \\
\hline Entre 16 y 20 años & 63 & $16.4 \%$ \\
\hline Entre 21 y 25 años & 39 & $10.2 \%$ \\
\hline Entre 26 y 30 años & 44 & $11.5 \%$ \\
\hline Entre 31 y 35 años & 13 & $3.4 \%$ \\
\hline Entre 36 y 39 años & 25 & $6.5 \%$ \\
\hline Total & 383 & $100.0 \%$ \\
\hline
\end{tabular}

Fuente: elaboración propia.

El 33\% de los emprendedores ha obtenido incrementos el año anterior por encima del promedio de la industria; el 33.4\% manifiesta no haber experimentado cambios en su desempeño, lo que es perjudicial en tanto no recupera lo perdido por inflación y crecimiento de la población; finalmente, el $33 \%$ ha tenido decrementos - solo $1.5 \%$ mayores al $20 \%$ -

Tabla 4. Desempeño de la empresa

\begin{tabular}{|lcc|}
\hline $\begin{array}{c}\text { Desempeño de la empresa } \\
\text { (incrementos, igual y decrementos) }\end{array}$ & Número & $\begin{array}{c}\text { Participación } \\
\text { relativa }\end{array}$ \\
\hline Decrecimientos de $20 \%$ o más & 2 & $0.5 \%$ \\
\hline Decrecimientos de $20 \%$ & 4 & $1.0 \%$ \\
\hline Decrecimientos hasta $20 \%$ & 122 & $31.9 \%$ \\
\hline No tuvieron cambios & 128 & $33.4 \%$ \\
\hline Incrementos hasta $20 \%$ & 85 & $22.2 \%$ \\
\hline Incrementos mayores a $20 \%$ & 42 & $11.0 \%$ \\
\hline Total & 383 & $100 \%$ \\
\hline
\end{tabular}

Fuente: elaboración propia. 
El desempeño atribuido a las prácticas gerenciales muestra que el 92.7\% de las empresas tiene hasta dos directivos (tabla 5). Ello reafirma su carácter de micro y pequeñas empresas con limitaciones para desarrollar prácticas gerenciales tales como el aprovechamiento de los resultados de investigación, el uso de $\mathrm{I}+\mathrm{D}+\mathrm{i}$ como ventaja competitiva y una limitación en su capacidad de acción (Schumpeter, 1939; McClelland \& Winter, 1970; Drucker, 1984; Guzmán \& Liñán, 2005; Pereira, 2007; González, 2007). Además, estos emprendedores no son ejecutivos con capacidad de delegación que persigan la eficacia en el mercado (González, 2007).

Tabla 5. Número de directivos

\begin{tabular}{|lcc|}
\hline \multicolumn{1}{|c|}{ Número de directivos } & Número & $\begin{array}{c}\text { Participación } \\
\text { relativa }\end{array}$ \\
\hline 1 directivo, incluido el propietario & 198 & $51.7 \%$ \\
\hline 2 directivos & 157 & $41.0 \%$ \\
\hline 3 directivos & 20 & $5.2 \%$ \\
\hline 4 directivos & 5 & $1.3 \%$ \\
\hline 5 directivos & 3 & $0.8 \%$ \\
\hline Total & 383 & $100.0 \%$ \\
\hline
\end{tabular}

Fuente: elaboración propia.

Se analizaron los coeficientes de correlación de las variables edad, sexo, edad de la empresa y número de empleados; no son significativas en un nivel 0.01 respecto a beneficios. Las variables de prácticas gerenciales significativas respecto a beneficios en un nivel 0.01 son las siguientes: acudir a préstamos externos, reinvertir beneficios generados, mantener grandes saldos en caja, desarrollar nuevos productos, trabajar en la construcción de marca, trabajar en un sistema claro de premios y castigos con todos los empleados, medir el desempeño de los empleados, usar publicidad para diferenciar los productos de la competencia, realizar publicidad a sus productos, hacer énfasis en la calidad de los productos, vender a un grupo definido de clientes, hacer énfasis en cantidad más que calidad, ofrecer garantías por largos periodos a sus consumidores, ejercer control sobre los canales de distribución, mantener altos niveles de inventarios de productos terminados, depender de un solo proveedor, mejorar la productividad de los empleados, predecir las tendencias de la industria, fijar objetivos de la empresa, desarrollar planes para la empresa, documentar los aspectos del plan, revisar el progreso de las actividades para determinar la consistencia del plan, el presupuesto y los pronósticos y asistir a ferias comerciales. 


\section{Control de cambios variables}

La variable dependiente es beneficios: incluidos todos los beneficios no monetarios recibidos como ingreso de la empresa, ¿han cambiado en el último año? La pregunta de beneficios tiene tres categorías: aumentaron, disminuyeron y no cambiaron.

Las variables independientes son logros, autonomía, ambición, agresividad, igualdad, autoridad, creatividad, dinero, energía, prestigio, seguridad, religión, placer, compasión, lealtad, confianza, competencias, riesgo, seguridad social, afecto, seguridad nacional, crecimiento, innovación, honestidad, responsabilidad, trabajo duro y optimismo.

\section{Descripción de las operaciones y análisis de los resultados de la encuesta}

Se creó la variable beneficios, que para este documento es la variable dependiente.

Tabla 6. Comportamiento de los beneficios

\begin{tabular}{|lccc|}
\hline $\begin{array}{l}\text { Incluidos todos los beneficios } \\
\text { no monetarios recibidos como } \\
\text { ingreso de la empresa }\end{array}$ & Frecuencia & $\%$ & \% Acumulado \\
\hline Aumentaron & 127 & 33.16 & 33.16 \\
\hline Disminuyeron & 128 & 33.42 & 66.58 \\
\hline No hubo cambios & 128 & 33.42 & 100.00 \\
\hline Total & 383 & 100.00 & \\
\hline
\end{tabular}

Fuente: elaboración propia.

Se hizo una prueba de independencia entre las variables independientes y la variable dependiente con la prueba de Chi-cuadrado para independencia. Esta prueba evalúa si las distribuciones de variables categóricas son diferentes una de la otra (se hace por par de variables). Acá casi todos los valores mostraron dependencia con excepción de placer, compasión y seguridad social, porque la probabilidad de la prueba Pearson - Chi-cuadrado es cercana a cero. 
Revista CIFE / ISSN: 0124-3551 e-ISSN: 2248-4914 / Bogotá-Colombia / Vol. 22 N. 37 / julio-diciembre 2020 / pp. $43-79$

Se rechaza la hipótesis nula de que las dos variables son independientes ${ }^{1}$. En las tablas 7 y 8 se encuentra el ejemplo de la salida de la variable sexo y valor logros, respectivamente.

Tabla 7. Comportamiento de los beneficios por sexo

\begin{tabular}{|lccc|}
$\begin{array}{l}\text { Incluidos todos los beneficios } \\
\text { no monetarios recibidos como } \\
\text { ingreso de la empresa }\end{array}$ & ¿Cuál es su sexo? & \\
\hline & Hombre & Mujer & Total \\
\hline Aumentaron & 71 & 56.00 & 127.00 \\
\hline Disminuyeron & 58 & 70.00 & 128.00 \\
\hline No hubo cambios & 70 & 58.00 & 128.00 \\
\hline Total & 199 & 184 & 383 \\
\hline
\end{tabular}

Pearson chi2 $(2)=3.4395 \quad \operatorname{Pr}=0.179$

Fuente: elaboración propia.

Tabla 8. Escala de valores de los empresarios de pymes

\section{Incluidos todos los beneficios los beneficios
no monetarios recibidos como ingreso de la empresa} corros

\begin{tabular}{|lcccccc|}
\hline Aumentaron & 24 & 53 & 36 & 8 & 6 & 127 \\
\hline Disminuyeron & 26 & 31 & 36 & 18 & 17 & 128 \\
\hline No hubo cambios & 14 & 55 & 39 & 9 & 11 & 128 \\
Total & 64 & 139 & 111 & 35 & 34 & 383 \\
\hline
\end{tabular}

Pearson $\operatorname{chi} 2(8)=22.2207 \quad \operatorname{Pr}=0.005$

\section{Escala de valores [Logros (realización)]}

$\begin{array}{cccccc}\text { Moderada } & \text { Extrema } & \text { Muy } & \text { Poco } & \text { No } & \text { Total } \\ \text { Importancia } & \text { importancia } & \text { importante } & \text { Importante } & \begin{array}{c}\text { importa } \\ \text { importan }\end{array} & \end{array}$


Como la variable dependiente beneficios es categórica con más de dos opciones, la mejor estimación que se puede hacer es través del método de Logit ordenado. Se hicieron varias regresiones, unas con todas las variables y otras con solo aquellas que resultaron dependientes. El que mejor se ajustó fue el que tenía las siguientes variables independientes: logros, autonomía, ambición, agresividad, igualdad, autoridad, lealtad, innovación, trabajo, honestidad ${ }^{2}$. El Chi-cuadrado reportado de ese modelo fue de 63.69 con un p-value de 0.01 , lo que indica que el modelo como un todo es estadísticamente significativo. Las variables que son estadísticamente significativas son logros, autonomía, ambición, agresividad, igualdad, autoridad, lealtad, innovación, honestidad y trabajo (tabla 9).

Tabla 9. Estadísticos

Iteration 0: $\log$ likelihood $=-420.76589$

Iteration 1: $\log$ likelihood $=-389.09699$

Iteration 2: $\log$ likelihood $=-388.92089$

Iteration 3: $\log$ likelihood $=-388.92066$

Iteration 4: $\log$ likelihood $=-388.92066$

\begin{tabular}{|cccc}
\hline Ordered logistic regression & Number of obs & $=383$ \\
& LR chi2(40) & $=$ & 63.69 \\
& Prob $>$ chi2 & $=$ & 0.0100 \\
\hline Log likelihood $=\mathbf{- 3 8 8 . 9 2 0 6 6}$ & Pseudo R2 & $=$ & 0.0757
\end{tabular}

\section{Beneficios}

\section{Logros}

$\begin{array}{lcccccc}\text { Extremadamente importante } & 0.385 & 0.326 & 1.18 & 0.237 & -0.253 & 1,024 \\ \text { Muy importante } & 0.802 & 0.329 & 2.44 & 0.015 & 0.158 & 1,447 \\ \text { Poco importante } & 0.617 & 0.414 & 1.49 & 0.136 & -0.194 & 1,428 \\ \text { No es importante } & 0.488 & 0.412 & 1.19 & 0.236 & -0.319 & 1,295\end{array}$

\section{Autonomía}

Extremadamente importante

0.304

0.347

0.88

0.381

$-0.376$

0.984 
Revista CIFE / ISSN: 0124-3551 e-ISSN: 2248-4914 / Bogotá-Colombia / Vol. 22 N.o 37 / julio-diciembre 2020 / pp. $43-79$

$\longrightarrow$ Continuación

\begin{tabular}{|c|c|c|c|c|c|c|}
\hline Beneficios & Coef. & St.Err. & t-value & p-value & \multicolumn{2}{|c|}{ [95\% Conf Interval] } \\
\hline Muy importante & -0.207 & 0.338 & -0.61 & 0.540 & -0.871 & 0.456 \\
\hline Poco importante & 0.356 & 0.395 & 0.90 & 0.368 & -0.419 & 1,130 \\
\hline No es importante & 0.257 & 0.405 & 0.63 & 0.526 & -0.537 & 1,052 \\
\hline \multicolumn{7}{|l|}{ Ambición } \\
\hline Extremadamente importante & 0.665 & 0.352 & 1.89 & 0.059 & -0.024 & 1,353 \\
\hline Muy importante & 0.002 & 0.344 & 0.01 & 0.996 & -0.673 & 0.676 \\
\hline Poco importante & 0.345 & 0.397 & 0.87 & 0.385 & -0.432 & 1,122 \\
\hline No es importante & 0.394 & 0.403 & 0.98 & 0.329 & -0.396 & 1,184 \\
\hline \multicolumn{7}{|l|}{ Agresividad } \\
\hline Extremadamente importante & -0.248 & 0.318 & -0.78 & 0.435 & -0.871 & 0.375 \\
\hline Muy importante & 0.415 & 0.310 & 1.34 & 0.181 & -0.193 & 1,023 \\
\hline Poco importante & -0.061 & 0.361 & -0.17 & 0.867 & -0.769 & 0.647 \\
\hline No es importante & -0.063 & 0.387 & -0.16 & 0.870 & -0.821 & 0.694 \\
\hline \multicolumn{7}{|l|}{ Igualdad } \\
\hline Extremadamente importante & -0.129 & 0.322 & -0.40 & 0.690 & -0.760 & 0.503 \\
\hline Muy importante & -0.145 & 0.315 & -0.46 & 0.646 & -0.762 & 0.473 \\
\hline Poco importante & 0.781 & 0.350 & 2.23 & 0.026 & 0.094 & 1,468 \\
\hline No es importante & -0.025 & 0.372 & -0.07 & 0.947 & -0.755 & 0.705 \\
\hline
\end{tabular}

\section{Autoridad}

\begin{tabular}{lcccccc} 
Extremadamente importante & -0.131 & 0.330 & -0.40 & 0.691 & -0.778 & 0.516 \\
\hline $\begin{array}{l}\text { Muy importante } \\
\text { Poco importante }\end{array}$ & -0.591 & 0.349 & -1.69 & 0.090 & $-1,276$ & 0.093 \\
\hline No es importante & -0.266 & 0.377 & -0.71 & 0.480 & $-1,006$ & 0.473 \\
\hline Lealtad & -0.202 & 0.390 & -0.52 & 0.605 & -0.966 & 0.563 \\
\hline $\begin{array}{l}\text { Extremadamente importante } \\
\text { Muy importante }\end{array}$ & 0.465 & 0.358 & 1.30 & 0.194 & -0.236 & 1,166 \\
\hline $\begin{array}{l}\text { Poco importante } \\
\text { M }\end{array}$ & 0.054 & 0.332 & 0.16 & 0.871 & -0.596 & 0.704
\end{tabular}


$\longrightarrow$ Continuación

\begin{tabular}{|c|c|c|c|c|c|c|}
\hline Beneficios & Coef. & St.Err. & t-value & p-value & \multicolumn{2}{|c|}{$[95 \%$ Conf Interval $]$} \\
\hline No es importante & 0.542 & 0.400 & 1.35 & 0.175 & -0.242 & 1,327 \\
\hline \multicolumn{7}{|l|}{ Innovación } \\
\hline Extremadamente importante & 0.341 & 0.325 & 1.05 & 0.294 & -0.296 & 0.977 \\
\hline Muy importante & -0.403 & 0.332 & -1.22 & 0.224 & $-1,053$ & 0.247 \\
\hline Poco importante & -0.204 & 0.386 & -0.53 & 0.597 & -0.961 & 0.552 \\
\hline No es importante & -0.135 & 0.379 & -0.36 & 0.721 & -0.877 & 0.607 \\
\hline \multicolumn{7}{|l|}{ Trabajo } \\
\hline Extremadamente importante & 0.127 & 0.348 & 0.36 & 0.716 & -0.556 & 0.810 \\
\hline Muy importante & 0.587 & 0.320 & 1.83 & 0.067 & -0.041 & 1,214 \\
\hline Poco importante & 0.595 & 0.428 & 1.39 & 0.165 & -0.244 & 1,434 \\
\hline No es importante & 0.620 & 0.348 & 1.78 & 0.075 & -0.062 & 1,303 \\
\hline \multicolumn{7}{|l|}{ Honestidad } \\
\hline Extremadamente importante & -0.325 & 0.341 & -0.95 & 0.340 & -0.993 & 0.343 \\
\hline Muy importante & -0.291 & 0.337 & -0.86 & 0.387 & -0.952 & 0.369 \\
\hline Poco importante & 0.080 & 0.397 & 0.20 & 0.841 & -0.698 & 0.857 \\
\hline No es importante & 0.567 & 0.425 & 1.33 & 0.182 & -0.266 & 1,400 \\
\hline cut 1 & 0.477 & 0.659 & & & -0.815 & 1,769 \\
\hline cut2 & 2,059 & 0.667 & & & 0.751 & 3,366 \\
\hline
\end{tabular}

Fuente: elaboración propia.

Finalmente, las predicciones de los posibles valores que tendría el modelo para cada una de las posibles categorías de la variable dependiente aumentaron = plologit, disminuyeron $=$ p2ologit, no hubo cambios $=$ p3ologit, y esos valores son muy parecidos a aquellos que se obtuvieron en las encuestas (tabla 10). 
Tabla 10. Resumen estadístico

\begin{tabular}{|cccccc} 
Variable & Obs & Mean & Std. Dev. & Mín & Máx \\
\hline plologit & 383 & 0.3404955 & 0.1649307 & 0.040366 & 0.8617038 \\
\hline p2ologit & 383 & 0.3329949 & 0.0530522 & 0.1063377 & 0.3759457 \\
\hline p3ologit & 383 & 0.3265096 & 0.1600568 & 0.0319586 & 0.8302278 \\
\hline
\end{tabular}

\section{Discusión}

Existen algunos modelos que se aproximan a la medición en contextos industriales que distintos países aplican actualmente. Para la gran industria se creó el instrumento 2010 Manufacturing Survey Instrument (Centre for Economic Performance, 2015; Delis \& Tsionas, 2018). Blackman (2003) analiza en conjunto el desempeño de las pymes a partir de las características propias, las características atribuidas, los valores y expectativas, y las prácticas gerenciales. En este documento se explica la parte del modelo de los valores y el desempeño en la industria de Bogotá. No se incluyen todos los previstos por este autor dados los diferentes contextos en que se recabó la información.

Los valores escogidos en este documento son logros, autonomía, ambición, agresividad, igualdad, autoridad, lealtad, innovación, trabajo y honestidad. Los valores con más peso encontrados por Blackman (2003) en Australia fueron poder, responsabilidad y afecto. Las prácticas gerenciales más destacadas y relacionadas con los valores son las siguientes: desarrollar nuevos productos, trabajar en un sistema claro de premios y castigos con todos los empleados, medir el desempeño de los empleados, hacer énfasis en cantidad más que calidad, mejorar la productividad de los empleados, predecir las tendencias de la industria, fijar objetivos de la empresa y desarrollar planes para la empresa.

En Colombia distintas organizaciones públicas y privadas están desarrollando proyectos que contribuyan a fortalecer las capacidades gerenciales de las empresas. El DNP y el DANE están consolidando una base de información de la gestión empresarial a través de un módulo en la Encuesta de Desarrollo e Innovación Tecnológica (CPC, 2018). Esta información permitirá construir un índice de los determinantes de la productividad que incluirá las condiciones de los habilitantes, el capital humano, los mercados y el ecosistema de innovación, considerados como factores necesarios para aumentar la productividad agregada y acelerar el desarrollo del país (CPC, 2018). 
El Ministerio de Comercio, Industria y Turismo implementará en varias regiones del país un programa llamado Fábricas de Productividad para garantizar que la Política de Desarrollo Productivo apoye a empresas de todos los sectores, se financie con recursos tanto nacionales como del Banco Mundial, y promueva el desarrollo de una oferta tecnológica a través de proyectos con agentes del ecosistema de innovación y su posterior transferencia al sector productivo (CPC, 2018).

\section{Conclusiones}

Con una muestra de 383 pymes del sector industrial de Bogotá se analizaron las características del emprendedor, sus valores y prácticas gerenciales expresados en los beneficios obtenidos. Los valores analizados y con estadísticos significativas fueron logros, autonomía, ambición, agresividad, igualdad, autoridad, lealtad, innovación, honestidad y trabajo. El desempeño expresado en obtención de beneficios por el desarrollo de la actividad empresarial en la industria bogotana está repartido entre quienes crecieron (33\%), no tuvieron cambios $(33.4 \%)$, y quienes han tenido decrementos $(33,4 \%)$. Los valores escogidos para este modelo son estadísticamente significativos.

\section{Referencias}

Amichai-Hamburger, Y. (2008). Potential and Promise of online volunteering. Computers in Human Behaviour, 24(2), 544-562. DoI: https://doi.org/10.1016/j.chb.2007.02.004

Amit, R., \& Zott, C. (2001). Value Creation in E-Business. Strategic Management Fournal, 22(6-7), 493 - 520. DOI: https://doi.org/10.1002/smj.187

Anderson, B., Salvucci, G., Ruane, A., Roads, J., \& Kanamitsu, M. (2008). A New Metric for Estimating the Influence of Evaporation on Seasonal Precipitation Rates. Fournal of Hydrometeorology, 9(3), 576-588. DOI: https://doi.org/10.1175/2007JHM968.1.

Aryee, S., Budhwar, P., \& Chen, Z. (2002). Trust as a mediator of the relationship between organizational justice and work outcomes: Test of a social exchange model. Fournal of Organizational Behavior, 23(3), 267 - 285. DOI: https://doi.org/10.1002/ job. 138

Asociación Nacional de Empresarios de Colombia (ANDI). (2018). Balance 2017, perspectivas 2018. Bogotá: ANDI. 
Revista CIFE / ISSN: 0124-3551 e-ISSN: 2248-4914 / Bogotá-Colombia / Vol. 22 N.o 37 / julio-diciembre 2020 / pp. $43-79$

Asociación Nacional de Empresarios de Colombia (ANDI). (2019). Colombia: Balance 2018 y Perspectivas 2019. Bogotá: ANDI.

Atkinson, J. W. (1957). Motivational determinants of risk-taking behavior. Psychological Review, 64(6, Pt.1), 359-372. DOI: https://doi.org/10.1037/h0043445

Avolio, B. J., \& Bass, B.M. (1991). The Full range of Leadership Development: Basic and Advanced Manuals. New York: Bass, Avolio \& Associates.

Avvisati, F., Jacotin, G., \& Vincent-Lancrin, S. (2013). Educating Higher Education Students for Innovative Economies: What International Data Tell Us. Turning Journal for Higher Education, 1(1), 223-240. DOI: https://doi.org/10.18543/ tjhe-1(1)-2013pp223-240

Backes, D. S., Koerich, M. G., \& Erdmann, A. L. (2007). Humanizando el cuidado a través de la valorización del ser humano: resignificación de los valores y principios por los profesionales de salud. Revista Latino-Americana de Enfermagem, 15(1), 34-41. DOI: https://doi.org/10.1590/S0104-11692007000100006

Banco de la República. (2018). Informe de la Junta Directiva al Congreso de la República. Bogotá: Banco de la República.

Banco de la República. (2019). Boletín de indicadores económicos. Bogotá: Banco de la República.

Banco Mundial. (2019). Colombia: panorama general. Banco Mundial. Recuperado de https:// www.bancomundial.org/es/country/colombia/overview

Barney, J. \& Hansen, M. (1994). Trustworthiness as a Source of Competitive Advantage, Strategic Management fournal, 15(S1), 175-190. DOI: https://doi.org/10.1002/ smj.4250150912

Bartz, W. et al. (1990). German tests. Level 1. Form A. Indianapolis, Indiana: Department of Education.

Bass, B. (1985). Leadership and Performance Beyond Expectations. New York: The Free Press.

Bettinelli, L. A., Waskievicz, J. \& Erdmann, A. (2004). Humanização do cuidado no ambiente hospitalar. En L. Pessini \& L. Bertachini (Orgs.), Humanização e cuidados paliativos (pp. 87-99). Sao Paulo: Ediciones Loyola.

Blackman, A. (2003). Entrepreneurs; Interrelationships between their characteristics, values, expectations, management practices and SME performance. Brisbane: Griffith University. 
Blais, R. \& Toulouse, J. (1990). Les motivations des entrepreneurs: une étude empirique de 2278 fondateurs d'entreprises dans 14 pays. Revue internationale P.M.E., 3(3-4), 267-425. DOI: https://doi.org/10.7202/1007982arBlanchard. K., \& O’Connor, M. (1997). Administración por valores. Bogotá: Editorial Norma.

Bosma, N., \& Schutjens, V. (2007). Patterns of Promising Entrepreneurial Activity in European Regions. Journal of Economic and Social Geography, 98(5), 675-686. DOI: https://doi.org/10.1111/j.1467-9663.2007.00433.x

Bosma, N., \& Schutjens, V. (2009). Determinants of early-stage entrepreneurial activity in European regions; Distinguishing low and high ambition entrepreneurship. En D. Smallbone, H. Landström \& D. Jones (Eds.), Entrepreneurship and Growth in Local, Regional and National Economies (Chapter 3). Frontiers in European Entrepreneurship Research. DOI: https://doi.org/10.4337/9781849802369.00010

Botero-García, J., López-Castaño, H., Posada, C. E., Ballesteros-Ruiz, C., \& GarcíaGuzmán, J. (2015). Economía colombiana. Análisis de coyuntura. $\mathcal{N}^{\circ}{ }^{\circ}$ 1. Medellín: Universidad EAFIT.

Brenkert, G. (2010). The Limits and Prospects of Business Ethics. Business Ethics Quarterly, 20(4), 703-709. DOI: http://doi.org/10.5840/beq201020444

Burns, J. M. (1978). Leadership. New York: Harper \& Row.

Cámara de Comercio de Bogotá (CCB). (2017). Bogotá - Región cerró el 2016 con más de 694.000 empresas y establecimientos de comercio activos. CCB. Recuperado de www.ccb.org. co/Sala-de-prensa/Noticias-CCB/2017 /Febrero/Bogota-Region-cerro-el-2016con-mas-de-694.000-empresas-y-establecimientos-de-comercio-activos

Cámara de Comercio de Bogotá. (2019). Balance de la economía de la región BogotáCundinamarca 2018. Bogotá: Cámara de Comercio de Bogotá.

Cañadas, M. (1996). Como crear empresas rentables. Editorial: Gestión 2000.

Carter, D. A., Simkins, B. J., \& Simpson, W. G. (2003). Corporate Governance, Board Diversity, and Firm Value. The Financial Review, 38(1), 33-53. DOI: https://doi. org/10.1111/1540-6288.00034

Casadesus-Masanell, R., \& Ricart, J. E. (2007). Competing Through Business Models. IEsE Business School. Recuperado de http://www.iese.edu/research/pdfs/DI-0713-E. pdf 
Revista CIFE / ISSN: 0124-3551 e-ISSN: 2248-4914 / Bogotá-Colombia / Vol. 22 N. 37 / julio-diciembre 2020 / pp. $43-79$

Cassar, A. (2007). Coordination and Cooperation in local, random and small world networks: Experimental evidence. Games and Economics Behavior, 58(2), 209-230. DOI: https://doi.org/10.1016/j.geb.2006.03.008

Centre for Economic Performance. (2015). Methodology - World Management Survey. Recuperado de https://cdnstatic8.com/worldmanagementsurvey.org/wp-content/ images/2010/09/Manufacturing-Survey-Instrument.pdf .

Chell, E., \& Allman, K. (2003). Mapping the Motivations and Intentions of thecnology orientated entrepreneurs. RE्छDD Management, 33(2), 117-134. DOI: https://doi. org/10.1111/1467-9310.00287

Collins, O. \& Moore, D. (1970). The Organi-zation Makers: A Behavioral Study of Independent Entrepreneurs. New York: Appleton-Century-Crofts Inc.

Consejo Gremial Nacional. (2018). Reactivación económica 2018-2022. Bogotá: Consejo Gremial Nacional.

Consejo Privado de Competitividad (CPC). (2018). Informe Nacional de Competitividad 20182019. Bogotá: Zetta Comunicadores.

Cooper, A., \& Dunkelberg W (1981). A New Look at Business Entry. Experiences of 1805 Entrepreneurs. En E. D. Vesper (Ed.), Frontiers of Enterpreneurship Research: The Proceedings of the Babson Conference on Enterpreneurship Research (pp. 1-20), Wellesley: Babson College.

Corporación Financiera Colombiana (Corficolombiana). (2017). Perspectivas económicas Corficolombiana. Proyecciones 2018: Despacito. Bogotá: Corficolombiana.

Covin, J., \& Slevin, D. (1989). Strategic management of small firms in hostile and benign environments. Strategic Management fournal, 10(1), 75-87. DOI: https://doi. org/10.1002/smj.425010010

Cox, T., \& Blake, S. (1991). Managing cultural diversity, implications for organizational competitiveness. The Executive, 5(3), 45-56. DOI: https://doi.org/10.5465/ AME.1991.4274465

Davidsson, P. (1989). Entrepreneurship - And after? A study of growth willingness in small firms. Journal of Business Venturing, 4(3), 211-226. DOI: https://doi. org/10.1016/0883-9026(89)90022-0

Davidsson, P. (1995). Determinants of Entrepreneurial Intentions. Paper prepared for the RENT IX Workshop, Piacenza, Italy. Recuperado de https://eprints.qut.edu.au/2076/1/ RENT_IX.pdf 
Dean, G. C., Thibodeaux, M. S., Beyerlein, M., Ebrahimi, B., \& Molina, D. (1993). Corporate entrepreneurship and competitive aggressiveness: A comparison of U.S. firms operating in Eastern Europe or the Commonwealth of Independent States with U.S. firms in other high-risk environments. En s. B. Prasad (Ed.), Advances in International and Comparative Management (pp. 31-54). Greenwich, CT: JAI Press.

Delis, M., \& Tsionas, M. (2018). Measuring management practices. International fournal of Production Economics, 199, 65-77. DOI: https://doi.org/10.1016/j.ijpe.2018.02.006

Djebarni, R., \& Ebie, S. (201 1). Equality and Diversity Management: An Investigation into the Business Case of Equality and Diversity Practice in sMEs. The International fournal of Diversity in Organizations, 10(5), 145-168. DOI: https://doi.org/10.18848/1447-9532/ CGP/v10i05/39907

Departamento Nacional de Planeación (DNP). (2013). Comisión nacional de competitividad, ciencia, tecnología e innovación. Bogotá: DNP.

Departamento Nacional de Planeación (DNP). (2017). Productividad y competitividad en Colombia: Retos y oportunidades. Bogotá: DNP.

Drucker, P. (1954). The practice of management. U.K.: Harper Collins Publishers.

Drucker, P. (1984). La innovación y el empresario innovador. Bogotá: Norma.

Eccles, J. S., \& Roeser, R. W. (2009). Schools, academic motivation, and stage-environment fit. En R. M. Lerner \& L. Steinberg (Eds.), Handbook of adolescent psychology: Individual bases of adolescent development (p. 404-434). John Wiley \& Sons Inc. DOI: https://doi.org/10.1002/9780470479193.adlpsy001013

Edelman, L., Brush, C., Manolova, T., \& Greene, P. (2010). Start -up Motivations and Growth Intentions of Minority Nascent Entrepreneurs. Fournal of Small Business Management, 48(2), 174-196. DOI: https://doi.org/10.1111/j.1540-627X. 2010.00291.x

Elenkov, D. (2002). Erratum to "Effects of leadership on organizational performance in Russian companies" [J. Bus. Res. 55(6) (2002) 467-480]. Fournal of Business Research, 56(3), 241. DOI: https://doi.org/10.1016/S0148-2963(02)00488-5

Engle, R., Schlaegeol, C., \& Dimitriadi, N. (2011). Institutions and entrepreneurial intent: a cross-country study. Fournal of Developmental Entrepreneurship, 16(2), 227-250. DOI: https://doi.org/10.1142/S1084946711001811

Fayol, H. (2003). Administración industrial y general. Bogotá: Stilo impresores. 
Revista CIFE / ISSN: 0124-3551 e-ISSN: 2248-4914 / Bogotá-Colombia / Vol. 22 N.o 37 / julio-diciembre 2020 / pp. $43-79$

Felin, T., Foss, N. J., Heimeriks, K. H., \& Madsen, T. L. (2012). Microfoundations of routines and capabilities: individuals, processes, and structure. Fournal of Management Studies, 49(8), 1351-1374. DOI: http://dx.doi.org/10.1111/j.1467-6486.2012.01052.x

Ferrier, W., Mac Fhionnlaoich, C., Smith, K., \& Grimm, C. (2002). The impact of performance distress on aggressive competitive behavior: A reconciliation of conflicting views. Managerial and Decision Economics, 23(4-5), 301-316. DOI: https://doi. org/10.1002/mde. 1067

Fontela, E., Guzmán, J., Pérez, M., \& Santos, F. J. (2006). The art of entrepreneurial foresight. Foresight, 8(6), 3-13. DOI: https://doi.org/10.1108/14636680610712496

Foti, R., \& Hauenstein, N. (2007). Pattern and Variable Approaches in Leadership Emergence and Effectiveness. The Fournal of Applied Psychology, 92(2), 347-55. DOI: https://doi.org/10.1037/0021-9010.92.2.347

Fundación para la Educación Superior y el Desarrollo (Fedesarrollo). (2019). Colombia es el segundo país que más crece en la región. Bogotá: Fedesarrollo.

Gasse, Y. (1982). Elaborations on the psychology of the entrepreneurship. Englewood Cliffs: Prentice-Hall.

Gibb, A. (1998). Educating tomorrow's entrepreneurs. Economic Reform Today, 4, 32-38.

Glaser, B., \& Strauss, A. (1967). The Discovery of Grounded Theory: Strategies for Qualitative Research. Mill Valley, CA: Sociology Press.

Global Entrepreneurship Monitor Colombia (GEM). (2017). Estudio de la actividad empresarial en 2017. Recuperado de http://gemcolombia.org/publications/

González, F. (2007). Creación de empresas. Madrid: Guía del emprendedor.

Guzmán, J. \& Santos, F. (1999). Hacia un modelo explicativo del empresario de calidad. Revista de Economía Industrial, 325, 133-150.

Guzmán, J., \& Liñán, F. (2005). Evolución de la educación empresarial en Estados Unidos y Europa: su papel como instrumento de desarrollo. Revista de Economía Mundial, 12, 149-171.

Hammond, T. R., \& Kleiner, B. H. (1992). Managing multicultural work environments. Equal Opportunities International, 11(2), 6. 
Hatchuel, A., \& Well, B. (2003). A new approach of innovation design: an introduction to C-K theory. En A. Folkeson, K. Gralen, M. Norell \& U. Sellgren (Eds.), The $14^{\text {th }}$ International Conference on Engineering Design (pp. 109-110). Recuperado de https:// www.researchgate.net/publication/228609791_A_new_approach_of_innovative_ design_an_introduction_to_CK_theory

Hermans, J., Vanderstraeten, J., van Witteloostuijn, A., Dejardin, M., Ramdani, D. \& Stam, E. (2015). Ambitious Entrepreneurship: A Review of Growth Aspirations, Intentions, and Expectations. Advances in Entrepreneurship, Firm Emergence and Growth, 17, 127-160. DOI: https://doi.org/10.1108/S1074-754020150000017011.

Hisrich, R., Langan-Fox, J., \& Grant, S. (2005). Entrepreneurship Research and Practice: A Call to Action for Psychology. American Psychologist, 62(6), 575-589. Dor: http://doi. org/10.1037/0003-066X.62.6.575

Illera, L. E., \& Illera, J. C. (2005). Politica empresarial. Linea de dirección y estrategias. Bogotá: Editorial CDSA. Recuperado de http://hdl.handle.net/10726/2428

Institute for Management Development (IMD). (2018). Anuario mundial de competitividad 2018. Sintesis de resultados para Colombia. Recuperado de https://colaboracion.dnp.gov. co/CDT/Desarrollo\%20Empresarial/Informe_IMD_2018.pdf

International Labour Office. (2007). Equality at work: Tackling the challenges. Recuperado de https://bit.ly/2BECFAG

Jöreskog, K., \& Sörborn, D. (1986). Lisrel vI: analysis of linear structural relationships by maximun likelihood instrumental variables and least squares methods. Mooresville, Indiana: Scientific Software.

Kang, H. (2005). A model of strategy formulation and implementation for service technology. Strategic Change, 14, 231-238. DOI: https://doi.org/10.1002/jsc.723

Kang, I., Jeon, S., Lee, S., \& Lee, C. K. (2005). Investigating Structural Relations Affecting the Effectiveness of Service Management. Tourism Management, 26(3), 301310. DOI: https://doi.org/10.1016/j.tourman.2004.02.006

Kantis, H. (Ed.). (2004). Desarrollo Emprendedor. América Latina y la experiencia internacional. Banco Interamericano de Desarrollo (BID). Recuperado de https://publications.iadb. org/es/publicacion/16310/desarrollo-emprendedor-america-latina-y-la-experiencia-internacional

Kiefer, K. (2005). Genome evolution among cruciferous plants: A lecture from the comparison of the genetic maps of three diploid species -Capsella rubella, Arabidopsis 
Revista CIFE / ISSN: 0124-3551 e-ISSN: 2248-4914 / Bogotá-Colombia / Vol. 22 N.o 37 / julio-diciembre 2020 / pp. $43-79$

lyrata subsp. petraea, and A. thaliana. American fournal of Botany 92(4), 761-767. DOI: https://doi.org/10.3732/ajb.92.4.761

Kirzner, I. (1986). The Open-Endedness of Knowledge. Ideas on Liberty. Recuperado de https:/ / fee.org/articles/the-open-endedness-of-knowledge/

Knight, F. (1942). Profit and Entrepreneurial Functions. The fournal of Economic History, 2, 126-132.

Koontz, H., \& Weihrich, H. (2003). Administración una perspectiva global (12a ed.). México: Mc Graw Hill.

Kotter, J., \& Heskett, J. (1995). Cultura de empresa y rentabilidad. Madrid: Ediciones Díaz de Santos.

Kouzes, J., \& Posner, B. (2002). The Leadership Challenge. California: Jossey-Bass.

Krueger, N., \& Carsrud, A. (1993). Entrepreneurial intentions: Applying the theory of planned behaviour. Entrepreneurship \& Regional Development, 5(4), 315-330. DOI: https:// doi.org/10.1080/08985629300000020

Locke, K. (2001). Grounded Theory in Management Research. London: Sage Publications.

Lohn, S., Reis, C., Ribeirete, F., \& de Souza, Y. (2017). Organizational practices required for innovation: a study in an information technology company. Gest. Prod., 24(2), 22 1-235. DOI: http://dx.doi.org/10.1590/0104-530x2161-16

Lord, S. (2006). Shared Values mean Shared Success. sz Business, $20(4), 56$.

Lowe, K. \& Gardner, W. (2000). Ten years of the leadership Quarterly Contributions and Challenges for the Future. The Leadership Quiartely, 11(4), 459-514. DOI: http:// doi.org/10.1016/S1048-9843(00)00059-X

Lowe, K. B., Kroeck, K. G., \& Sivasubramaniam, N. (1996). Effectiveness correlates of transformational and transactional leadership: A meta-analytic review of the MLQ literature. The Leadership Quarterly, 7(3), 385-425. DOI: http://dx.doi.org/10.1016/ S1048-9843(96)90027-2.

Lumpkin, G. \& Dess, G. (1996). Clarifying the Entrepreneurial Orientation Construct and Linking It to Performance. The Academy of Management Review, 21(1), 135-172. DOI: https://doi.org/10.2307/258632

Luxemburg, R. (2003). The Accumulation of Capital Rare Masterpieces of Philosophy and Science. Routledge. 
Magretta, J. (2002). Why Business Models Matter. Harvard Business Review, 80(5), 86-92.

Marx. K. (1984). El Capital. Crítica de la economía politica. México: Fondo de Cultura Económica.

Mayer, R., Davis, J. \& Schoorman, D. (1995). An Integrative Model of Organizational Trust. The Academy of Management Review, 20(3), 709-734. DOI: http://doi. org $/ 10.2307 / 258792$

Mayer, K. B., \& Goldstein, S. (1961). The First Two Years: Problems of Small Firm Growth and Survival. Washington, D.C.: U.s. Government Printing Office.

McCann, J., Sparks, B., \& Kohntopp, T. (2017). Leadership Integrity and Diversity in the Workplace. Research in Economics and Management, 2(5), 177-191. Dor: https://doi. org/10.22158/rem.v2n5p177

McClelland, D. (1989). Estudio de la motivación humana. Madrid: Narcea Ediciones.

McClelland, D., \& Winter, D. (1970). Cómo se motiva el éxito económico. Ciudad de México: Unión tipográfica editorial hispanoamericana.

McDonald, P., \& Gandz, J. (1992). Getting value from shared values. Organizational Dynamics, 20(3), 64-77. DoI: https://doi.org/10.1016/0090-2616(92)90025-I

McEnrue, M. (1993). Managing diversity: Los Angeles before and after the riots. Organizatinal Dynamics, 21(3), 18-29. DOI: https://doi.org/10.1016/00902616(93)90068-G

Monga, M. (2016). Integrity and its antecedents: A unified conceptual framework of integrity. The Journal of Developing Areas, 50(5), 415-421. Dor: https://doi.org/10.1353/ jda.2016.0040

Mouján, H. (2006). Cómo desarrollar microemprendimientos. Buenos Aires: Longseller.

Ng, K. Y., Ang, S., \& Chan, K. Y. (2008). Personality and leader effectiveness: A moderated mediation model of leadership self-efficacy, job demands, and job autonomy. Fournal of Applied Psychology, 93(4), 733-743. DOI: https://doi.org/10.1037/00219010.93.4.733

Nickels, W., McHugh, J., \& McHugh, S. (1997). Introducción a los negocios. Madrid: Mc Graw Hill.

Nonaka, I., \& Takeuchi, H. (2008). The Wise Company: How Companies Create Continuous Innovation. New York: Oxford University Press. 
Revista CIFE / ISSN: 0124-3551 e-ISSN: 2248-4914 / Bogotá-Colombia / Vol. 22 N. 37 / julio-diciembre 2020 / pp. $43-79$

Nonaka, I., \& Von Krogh, G. (2009). Tacit Knowledge and Knowledge Conversion: Controversy and Advancement in Organizational Knowledge Creation Theory. Organization Science, 20(3), 635-652. DOI: http://doi.org/10.1287/orsc. 1080.0412

Nooteboom, B., Berger, H., \& Noorderhaven, N. (1997). Effect of Trust and Governance on Relational Risk. The Academy of Management fournal, 40(2), 308-338. DOI: http:// doi.org/10.2307/256885

Nueno, P. (1994). Emprendiendo. El arte de crear empresas y sus artistas. Bilbao: Ed. Deusto.

Nuevo, P. (2009). Emprendiendo la destrucción creativa. Harvard Deusto Business Review, 181. Recuperado de https://www.harvard-deusto.com/emprendiendo-la-destruccion-creativa

Organización para la Cooperación y el Desarrollo Económicos (OCDE). (2017). Estudios Económicos de la OCDE Colombia. Bogotá: OCDE.

Ogalla, F. (2005). Sistema de gestión: una guía práctica. Madrid: Editorial Diaz de Santos

Paelmke, H., \& Erwee, R. (2008). Diversity management in German and Indian manufacturing companies. International fournal of Organizational Behaviour, 13(1), 28-51.

Palanski, M., Gullen, K., Gentry, W., \& Nichols, C. (2015). Virtuous Leadership: Exploring the Effects of Leader Courage and Behavioral Integrity on Leader Performance and Image. Fournal of Business Ethics, 132(2), 297-310. DOI: https://doi. org/10.1007/s10551-014-2317-2

Pereira, F. (2007). La Evolución del Espíritu Empresarial como campo del conocimiento. Hacia una visión sistémica y humanista. Cuadernos de Administración, 20(34), 11-37.

Ponti, F., \& Ferrás, X. (2008). Passion for Innovation. Bogotá: Norma Editorial.

Prat, S. (1986). El emprendedor hoy. Madrid: Ed. Hispano Europea.

Ramírez, P., Noel, D., \& Cabello, M. (1997). Empresas competitivas. México: McGraw-Hill.

Reynolds, P., Bygrave, W. D., Autio, E., Cox, L. W., \& Hay, M. (2002). Global Entrepreneurship Monitor: 2002 Executive Report. DOI: http://doi.org/10.13140/RG.2.1.1977.0409

Riggio, R. E., Riggio, H. R., Salinas, C., \& Cole, E.J. (2003). The role of social and emotional communication skills in leader emergence and effectiveness. Group Dynamics: Theory, Research, and Practice, 7(2), 83-103. DOI: https://doi.org/10.1037/1089-2699.7.2.83

Robbins, S., \& Coulter, M. (2005). Administración. Ciudad de México: Pearson Educación México. 
Rokeach, M., (1973). The nature of human values. New York: The Free Press.

Rosenblatt, L. (1985). Viewpoints: Transacion versus interaction a terminological rescue operation. Research in the Teaching of English, 19(1), 96-107.

Ross, R., \& Schneider, R. (1992). From equality to diversity: a business case for equal opportunities. London: Pitman.

Salahudin, S. N., Alwi, M. N. R., Baharuddin, S. S., \& Halimat, S. S. (2016). The Relationship between Work Ethics and Job Performance. The European Proceedings of Social \& Behavioural Sciences EpsBS, 3rd International Conference on Business and Economics, 21 - 23 September, 2016.

Schumpeter, J. (1939). Ciclos económicos. Análisis teórico, histórico y estadístico del proceso capitalista. Madrid: Prensas universitarias de Zaragoza.

Schumpeter, J. (1946). Capitalismo, socialismo y democracia. Ed. Claridad, Buenos Aires.

Schumpeter, J. (1947). The creative response in economic history. The fournal of Economic History, 7(2), 149-159

Sexton, D., \& Bowman-Upton, N. (1987). Evaluation of an Innovative Approach to Teaching Entrepreneurship. Journal of Small Business Management, 25(1), 35-43. Recuperado de https://ssrn.com/abstract=1505256

Shapero, A. (1982). The Social Dimensions of Entrepreneurship. En C. A. Kent, D. L. Sexton \& K. H. Vesper (Eds.), Encyclopedia of Entrepreneurship (pp. 72-90). Berkeley: Prentice Hall. Recuperado de ssRN: https://ssrn.com/abstract=1497759

Siliceo, A. (1997). Lideres para el siglo XXI. Ciudad de México: Mc Graw Hill.

Stoycheva, K., \& Lubart, T. (2001). The Nature of Creative Decision Making. En C. M. Allowood \& M. Selart (Eds.) Decision Making: Social and Creative Dimensions (pp. 15-33). Dordrecht: Springer. DOI: https://doi.org/10.1007/978-94-015-9827-9_2

Taylor, F. (2003). Principios de la administración científica. Bogotá: Stilo impresores.

Thurik, R., Hessels, J., \& Gelderen, M. (2008). Entrepreneurial aspirations, motivations, and their drivers. Small Business Economics, 31, 323-339. DOI: https: / /doi.org/10.1007/ s1 1187-008-9134-x

Tominc, P., \& Rebemik, M. (2007). Growth aspirations and cultural support for entrepreneurship: A comparison of post socialist countries. Small Business Economics, 28, 239-255. DOI: https://doi.org/10.1007/s11187-006-9018-x 
Revista CIFE / ISSN: 0124-3551 e-ISSN: 2248-4914 / Bogotá-Colombia / Vol. 22 N.o 37 / julio-diciembre 2020 / pp. $43-79$

Trautwein, M., Wiegmann, B. M., Beutel, R., Kjer, K. M., \& Yeates, D. K. (2012). Advances in Insect Phylogeny at the Dawn of the Postgenomic Era. Annual Review of Entomology, 57(1), 449-468. DOI: https://doi.org/10.1146/annurev-ento-120710-100538

Unipymes. (4 de febrero de 2019). Bogotá - región cerró el 2018 con 764.639 empresas y establecimientos de comercio. Unipymes. Recuperado de https://www.unipymes. com/bogota-region-cerro-el-2018-con-764-639-empresas-y-establecimientos-decomercio/

Varela, R., Moreno, J., \& Bedoya, M. (2015). GEM Colombia 2006-2013. Report. Cali: Icesi.

Varona, F. (2002). Conceptualization and Management of Communication Satisfaction and Organizational Commitment in Three Guatemalan Organizations. American Communication fournal, 5(3).

Veciana, José (2001). Creación de empresas como campo de estudio y salida profesional: Evolución, estado actual y tendencias de desarrollo. RGE-Revista de Gestão e Economia. Universidade Da Beira Interior. Covilha, Portugal, pp. 2-9

Vrontis, D., \& Thrassou, A. (Eds.). (2013). Innovative Business Practices. Prevailing a Turbulent Era. Newcastle: Cambridge Scholars Publishing.

Weber, M. (1984). La ética protestante y el espiritu del capitalismo. Madrid: Sarpe.

Whetten, D., \& Cameron, K. (2011). Desarrollo de habilidades directivas (8 ${ }^{\mathrm{a}}$ ed.). México: Pearson.

Whitener, E., Brodt, S., Korsgaard, M., \& Werner, J. (1998). Managers as Initiators of Trust: An Exchange Relationship Framework for Understanding Managerial Trustworthy Behavior. The Academy of Management Review, 23(3), 513-530. DoI: https:// doi.org/10.2307/259292

Wigfield, A. (1994). Expectancy-value theory of achievement motivation: A developmental perspective. Educational Psychology Reviere, 6, 49-78. DoI: https://doi.org/10.1007/ BF02209024

Wigfield, A., \& Eccles, J. (2000). Expectancy-Value of Achievement Motivation. Contemporary Educational Psychology, 25(1), 68-81. DOI: http://dx.doi.org/ 10.1006/ ceps.1999.1015

Wong, W. (2005). Input Enhancement: From Theory and Research to the Classroom. New York: McGraw-Hill. 
Wong, Y.-T., Ngo, H.-Y., \& Wong, G.-S. (2002). Affective organizational commitment of workers in Chinese joint ventures. Fournal of Managerial Psychology, 17(7), 580-598. DOI: https://doi.org/10.1108/02683940210444049

Yunis, E. (2006). ¡Somos así! Bogotá: Editorial Bruna.

Yunis, E. (2009). ¿Por qué somos así? Bogotá: Temis.

Zarate, I. (2003). Valores, civismo familia y sociedad. Guanajuato: Rezza Editores

Zucker, L. (1986). Production of Trust: Institutional sources of economics structure, 1840-1920. Research in Organizational Behaviour, 8, 53-111. 